\title{
Bio-interface Polymerisation: Synthesis of Polyaniline on the Marine Algae Surface
}

\author{
Yuki Kudo, Hiromasa Goto*
}

Division of Materials Science, Faculty of Pure and Applied Sciences, University of Tsukuba, Tsukuba, Ibaraki 305-8573, Japan

E-mail: s-ykudo@ims.tsukuba.ac.jp (Y. Kudo), gotoh@ims.tsukuba.ac.jp (H. Goto)

Keywords: ESR; polyaniline; marine algae; SEM.

Abstract: Marine algae Gelidium, Grateloupia elliptica Holmes, and Codium fragile are employed for preparation of PANI/seaweed composites. Infrared absorption (IR), electron spin resonance (ESR) spectroscopy measurements are carried out to confirm the resultant structure. The direct use of natural biological materials for polymerisation reaction allows us to production of polymers with characteristic surface. This can be referred to as bio-interface polymerisation.

\section{Introduction}

Combination of conducting polymers and textile has been paid attention for production of conducting fibres and conducting films with good mechanical strength and flexibility. Polyaniline (PANI) is one of the most promising conducting polymers because synthesis of polyaniline is simple, and can perform in the water medium [1]. Furthermore, PANI shows good electrical conductivity in the doped (oxidised) form.

In this research, we attempt polymerisation of aniline as a monomer on the natural marine algae to obtain characteristic surface structure as a new attempt of bio-interface polymerisation with sea biological materials.

\section{Experiment}

\section{Seaweeds}

Seaweeds Gelidium [2], Grateloupia elliptica Holmes, and Codium fragile were obtained in Shimoda (Japan) with a support and instruction of diving by Shimoda Marine Research Centre, University of Tsukuba (Japan) on August, 2015.

Figure 1 show Gelidium (a), Grateloupia elliptica Holmes (b), and Codium fragile (c).

Polymerisation on the surface of seaweeds.

A solution of aniline and sulfuric acid in water was stirred in the presence of the marine alga (Gelidium, Grateloupia elliptica Holmes, or Codium fragile) for $1 \mathrm{~h}$ at $\mathrm{rt}$. The mixture was cooled to $0{ }^{0} \mathrm{C}$ and stirred for $1 \mathrm{~h}$. Then, ammonium peroxodisulfate (APS) was slowly added into the mixture (Table 1). After reaction for $24 \mathrm{~h}$, the resultant material was washed with a large volume of water, and large volume of methanol followed by filtration. The PANI/marine alga as bio-composites thus prepared was collected and dried under vacuum to afford a desired product. PANI was coated on the surface of the marine algae, as shown in Figure 1(d-f). The shape of the marine algae is remained after polymerisation. The bio-composites thus prepared are abbreviated as PANI-Gelidium, PANI-Grateloupia, and PANI-Codium. Surface of the marine algae is coated with PANI. The surface colour is emerald green. 


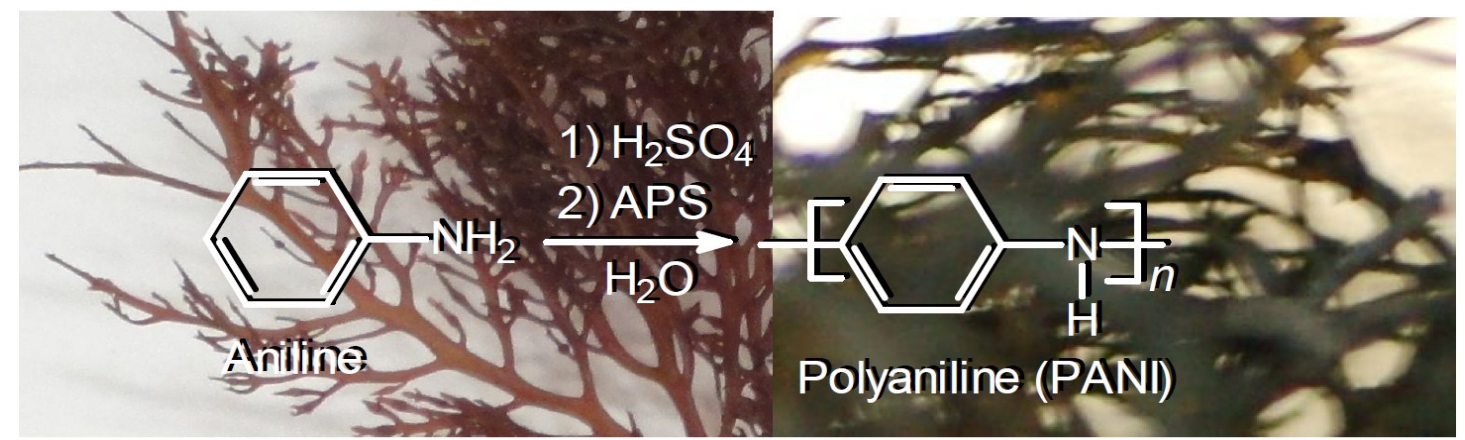

Scheme 1. Polymerisation of aniline in the presence of a marine alga. Background left: Gelidium, right: resultant PANI-Gelidium as a bio-composite.

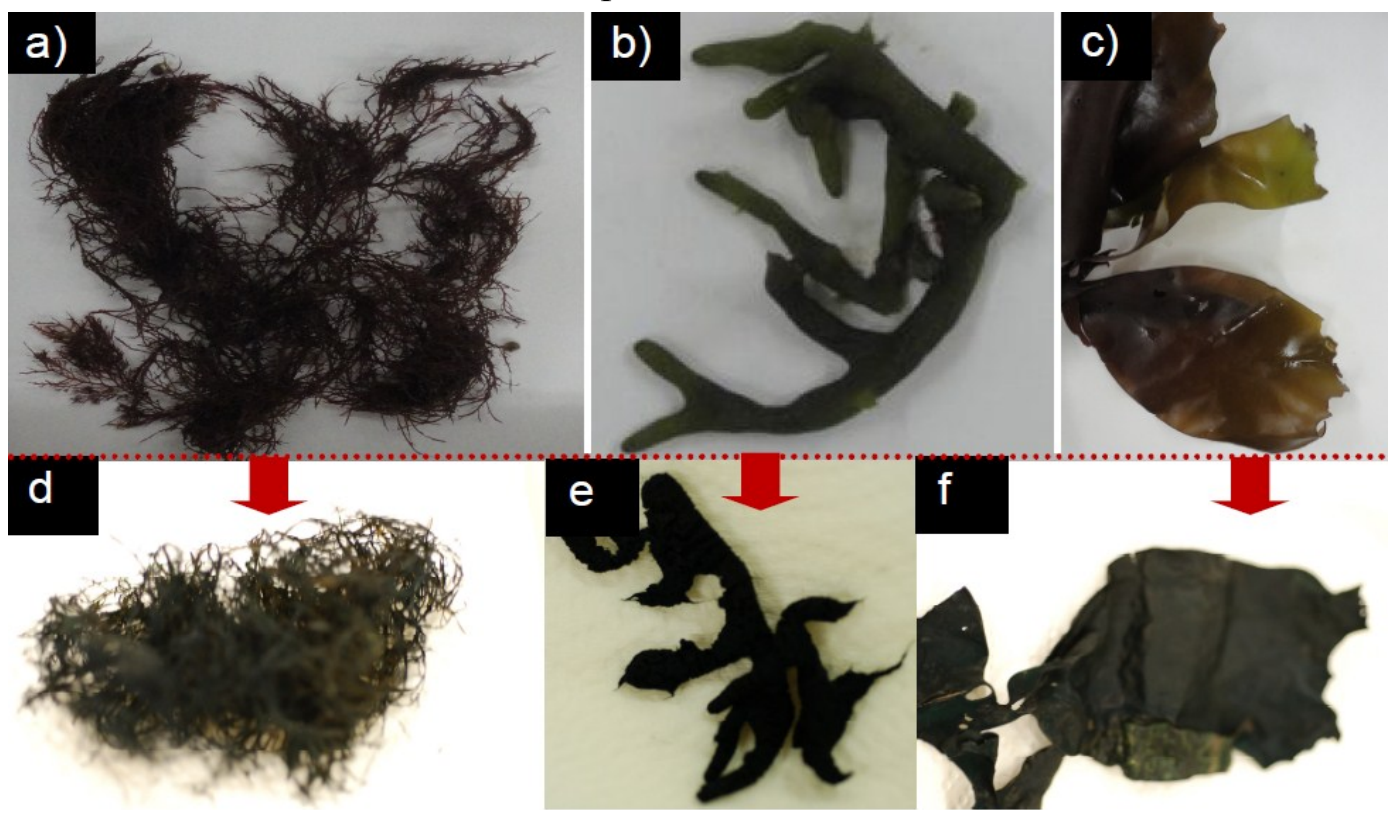

Figure 1. (a): Gelidium, (b): Codium fragile, (c): Grateloupia elliptica Holmes. (d): Bio-composite of PANI/Gelidium (PANI-Gelidium), (e): PANI/Codium fragile (PANI-Codium), (f): PANI/Grateloupia elliptica Holmes (PANI-Grateloupia).

SEM

Scanning electron microscopy (SEM) observations for the bio-composite were carried out. Figure 2(a,b) displays the SEM images of Gelidium, showing tube structure. Magnification image of the sample evaluates that the surface is flat. After polymerisation of aniline on the surface (Figure 2(c,d), the surface is covered with fine structure consisting of PANI.

Figure 3(a,b) shows surface of Codium fragile, showing crinkle structure. After formation of composite with PANI, the surface becomes smooth, as shown in Figure 3(c). Magnification image of the surface displays rocks like structure, as shown in Figure 3(d).

Table 1. Polymerisation of aniline, the ESR results and electrical conductivities for the resultant polymers.

\begin{tabular}{lcc|c}
\hline Bio-composite & $\begin{array}{c}\text { Aniline } \\
(\mathrm{g})^{\mathrm{a}}\end{array}$ & $\begin{array}{c}\text { Spin concentration } \\
(\mathrm{spin} / \mathrm{g})^{\mathrm{b}}\end{array}$ & $\begin{array}{c}\text { Electrical conductivity } \\
\left(\mathrm{S} / \mathrm{cm}^{-1}\right)\end{array}$ \\
\hline PANI-Gelidium & 3.060 & $1.81 \times 10^{22}$ & $1.85 \times 10^{-6}$ \\
PANI-Codium & 4.595 & $2.72 \times 10^{17}$ & $7.50 \times 10^{-3}$ \\
PANI-Grateloupia & 3.878 & $1.60 \times 10^{18}$ & $2.79 \times 10^{-2}$ \\
\hline
\end{tabular}

${ }^{\mathrm{a}}$ Quantity of aniline as a monomer in polymerisation. APS: $1 \mathrm{~g}, \mathrm{H}_{2} \mathrm{SO}_{4}: 0.5 \mathrm{~g}, \mathrm{H}_{2} \mathrm{O}: 200 \mathrm{~mL}$.

${ }^{\mathrm{b}} \mathrm{Spin}$ concentration of resultant polymers evaluated with the ESR. 


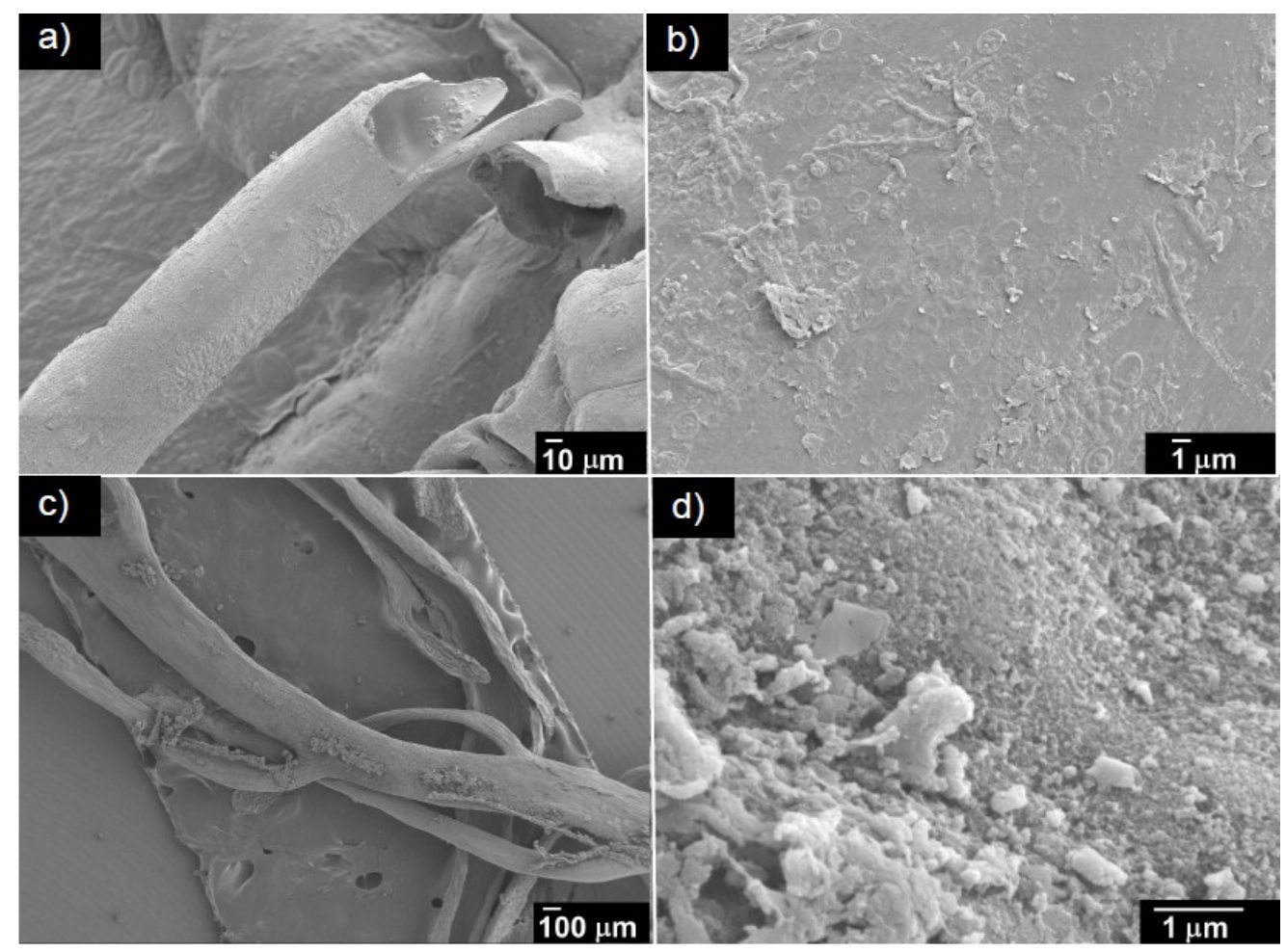

Figure 2. Scanning electron microscopy (SEM) images of a marine alga Gelidium (a,b). SEM image of the sample after deposition of PANI on the surface to form a bio-composite (PANI-Gelidium) (c,d).

Figure 4 shows surface images of Grateloupia elliptica Holmes and PANI/Grateloupia elliptica Holmes (PANI/Grateloupia). Grateloupia elliptica Holmes displays fine concavo-convex structure. After formation of the composite, the surface of the sample shows fine grooves. A magnification image evaluated that the surface partly shows a fibril like structure. This can be due to formation of PANI nano-fibre structure through self-aggregation of PANI [3].

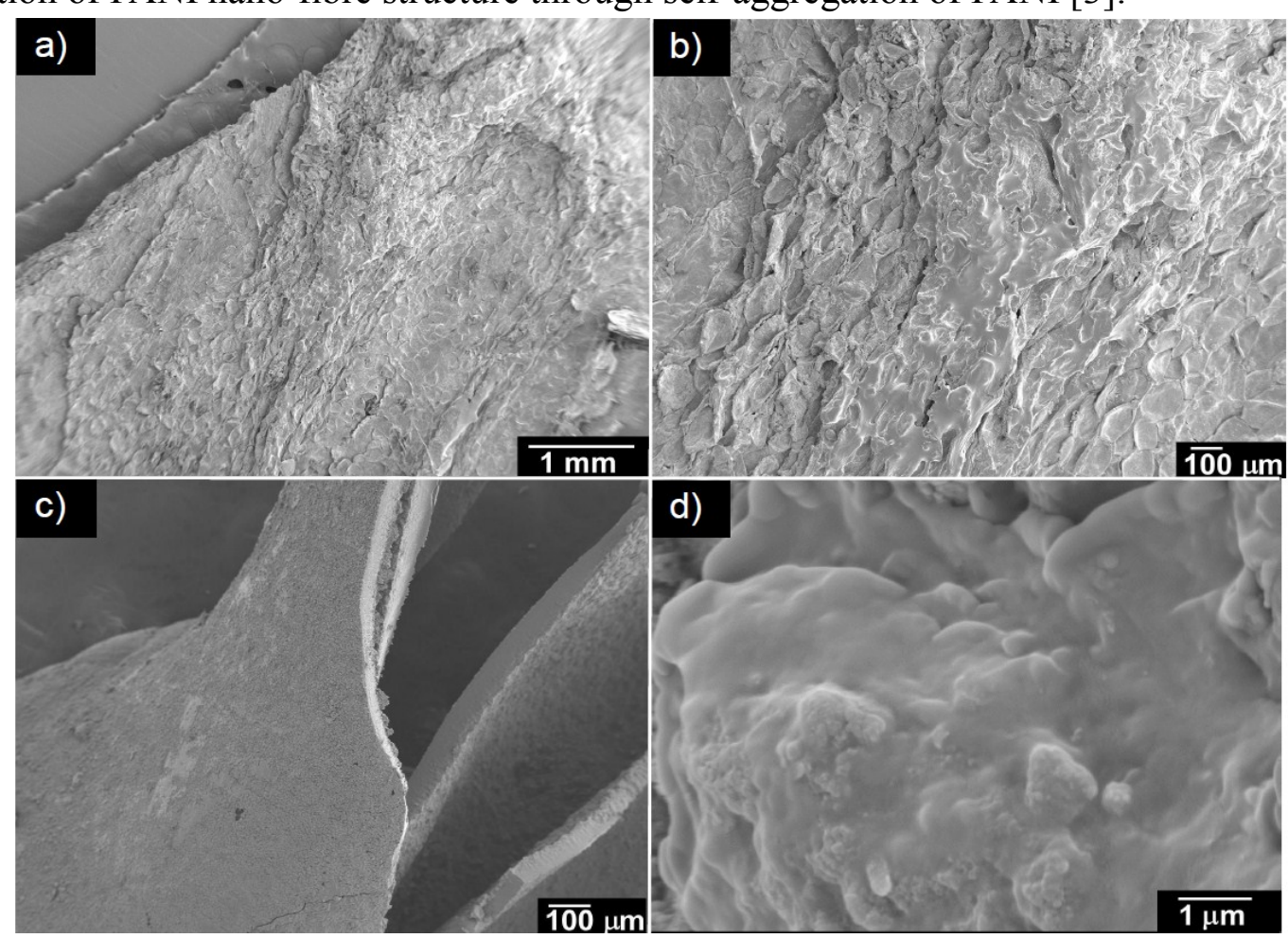

Figure 3. SEM images of a marine alga Codium fragile (a,b). SEM image of the sample after deposition of PANI on the surface to form a bio-composite (PANI-Codium) (c,d). 
IR

Infrared absorption (IR) spectroscopy measurements results are shown in Figure 5. The spectra shows characteristic absorption bands derived from PANI. Here, quinoid structure in the PANI is due to quinone-imine structure of the doped form (Figure 6). Benzenoid structure (B) is a form of neutral monomer repeat unit in the main-chain of the PANI. As prepared PANI is a half-doped form because the main-chain is doped with sulfuric acid during the polymerisation process. The stretching vibration of $\mathrm{C}=\mathrm{C}$ is observed at $1650 \mathrm{~cm}^{-1}$ for PANI-Gelidium. PANI-Codium and PANI- PANI-Grateloupia show weak absorption band at this range. An absorption band due to quinoid structure (Q) is observable at around $1540 \mathrm{~cm}^{-1}$. Absorption bands ascribed to quinoid-benzenoid-quinoid (Q-B-Q) stretching ( $\left.v_{\mathrm{QBQ}}\right)$ and benzenoid-benzenoid-benzenoid (B-B-B) stretching $\left(v_{\mathrm{BBB}}\right)$ vibrations are observed at $1300 \mathrm{~cm}^{-1}$ and $1251 \mathrm{~cm}^{-1}$, respectively.

These results indicates that the PANI layer coated on the marine algae is in the form of PANI-emeraldine salt [4].

\section{ESR}

Electron spin resonance (ESR) reveals unpaired electron of the sample. The bio-composite thus prepared is coated with the PANI layer. Figure 7 shows the ESR results of the bio-composites PANI-Gelidium, PANI-Codium, and PANI-Grateloupia. These composites show Lorenz type symmetrical resonance. PANI-Grateloupia displays narrow peak-to-peak line width $\left(\Delta H_{\mathrm{pp}}=0.72\right.$ $\mathrm{mT}$ ), indicating the charge carrier (polarons, radical cations [5]) is relatively delocalised along the main-chain (Figure 8).
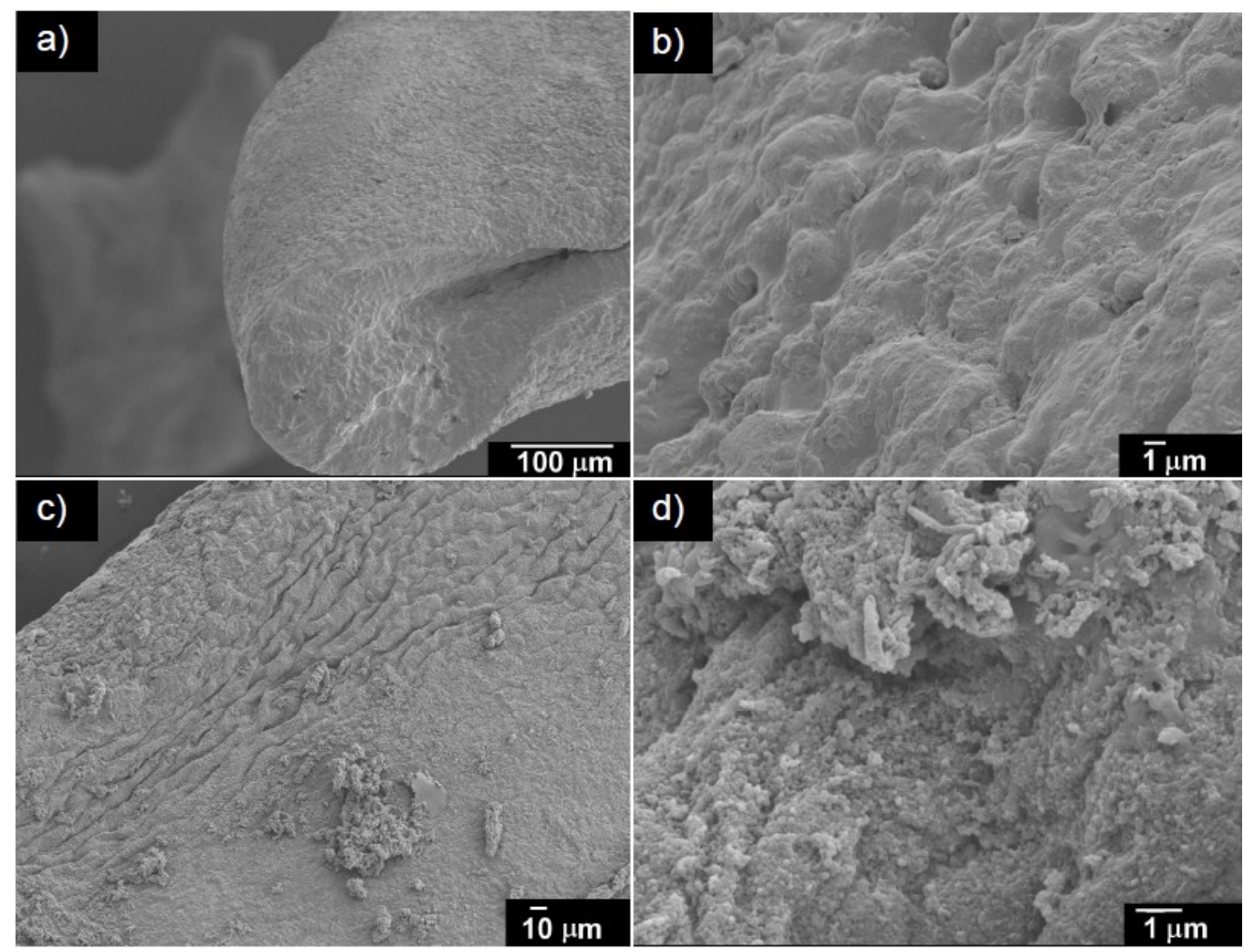

Figure 4. SEM images of a marine alga Grateloupia elliptica Holmes (a,b). SEM images of the sample after deposition of PANI on the surface to form a bio-composite (PANI-Grateloupia) (c,d). 
The ESR signal of PANI-Codium is small compared with other samples. This may be due to low amount deposition of the PANI on the marine alga in the polymerisation reaction. However, it shows narrow peak-to-peak line width $\left(\Delta H_{\mathrm{pp}}=0.46 \mathrm{mT}\right)$, indicating well delocalisation of polarons along the main-chain of PANI. In the case of the ESR spectrum exhibits asymmetry Dysonian lineshape, observable for graphite and nano-carbons, microwave can not reach inside of the sample due to skin-effect [6]. However, microwave from the ESR percolates inside of the thin layer of PANI in the samples to show the symmetry line shape for the bio-composite samples. PANI-Grateloupia shows further narrow peak-to-peak line width to be $0.24 \mathrm{mT}$.

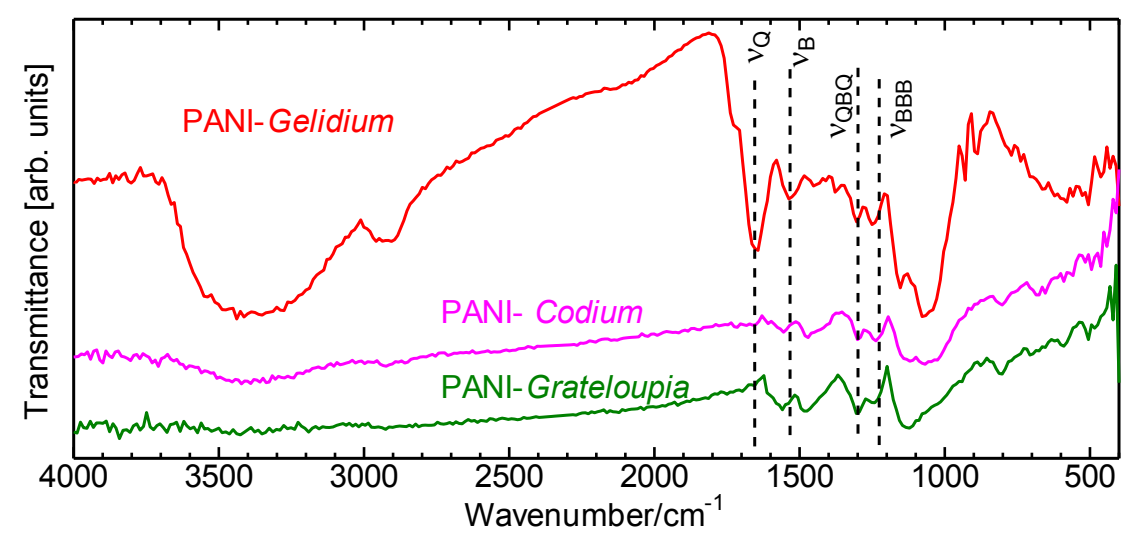

Figure 5. Infrared (IR) spectra of the resultant bio-composites Gelidium PANI (PANI-Gelidium), Codium fragile/PANI (PANI-Codium), and Grateloupia elliptica Holmes/PANI (PANI-Grateloupia).

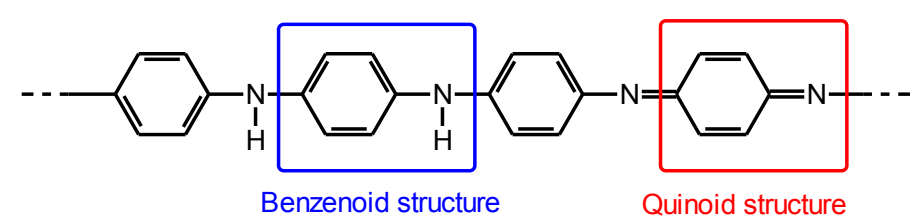

Figure 6. Benzenoid and quinoid structure of the PANI.

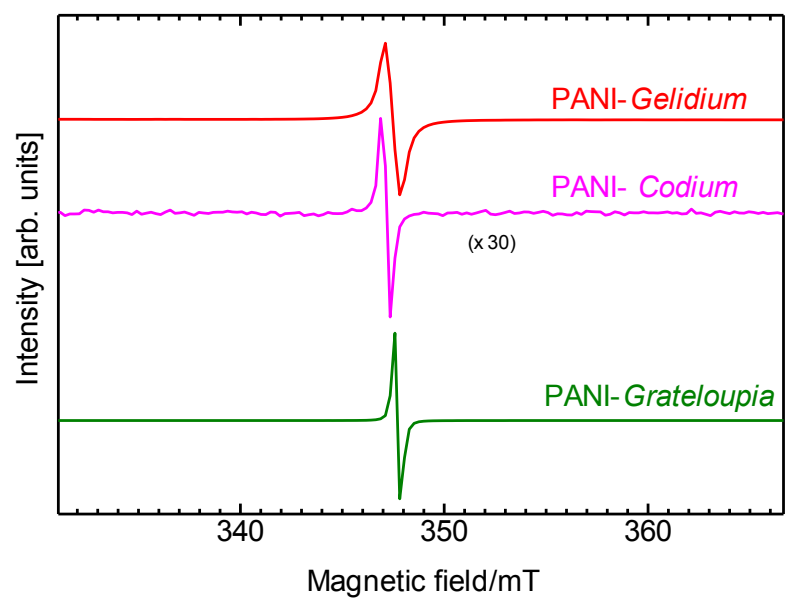

Figure 7. Electron spin resonance (ESR) spectroscopy measurement results.

Electrical conductivity

Electrical conductivities of bio-composites PANI-Gelidium, PANI-Codium, and PANI-Grateloupia were evaluated with a four-probe method. Conductivity of PANI-Gelidium is somewhat low $\left(1.9 \times 10^{-6} \mathrm{~S} / \mathrm{cm}\right)$ (Table 1). This is accordance with localisation of charge carrier of the PANI-Gelidium from the ESR result. PANI-Codium, and PANI-Grateloupia show good 
conductivity to be $7.5 \times 10^{-3}$ and $2.8 \times 10^{-2} \mathrm{~S} / \mathrm{cm}^{-1}$, respectively. These results indicate that the $\mathrm{PANI} /$ marine algae prepared in this study can be referred to as electrical conductive bio-composites. Figure 9 summerises electrical conductivity and the ESR results of the bio-composites, indicating the $\Delta H_{\mathrm{pp}}$ is reciprocal proportion tendency to the electrical conductivity.

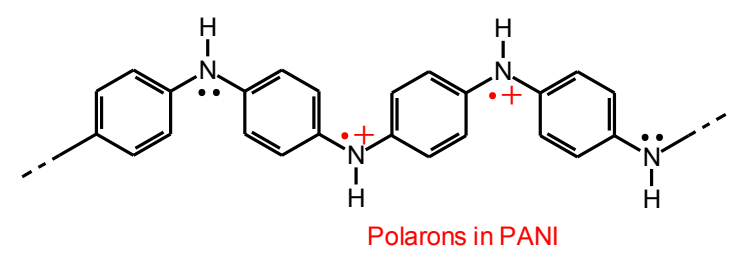

Figure 8. Polarons (radical cations) in PANI.

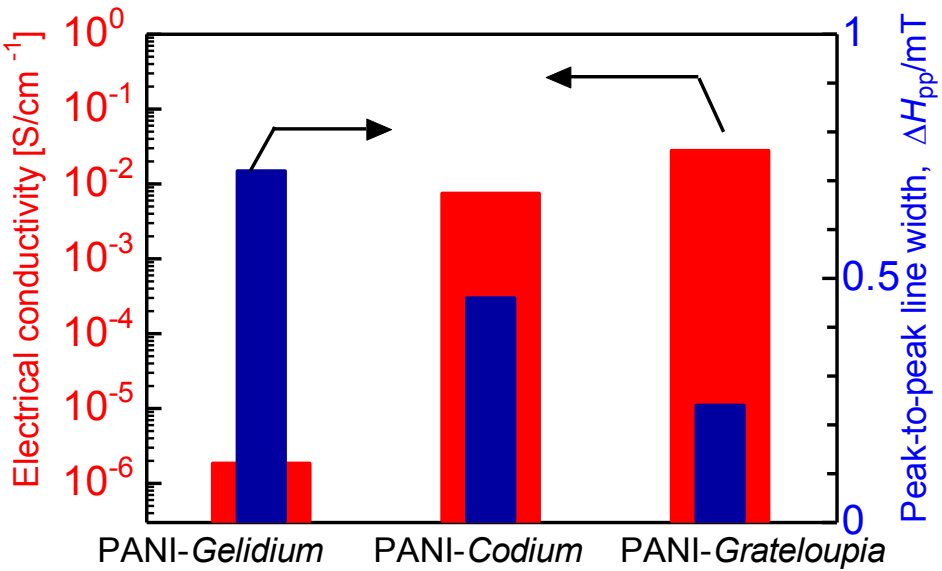

Figure 9. Electrical conductivity and peak-to-peak line width $\left(\Delta H_{\mathrm{pp}}\right)$ of the ESR signal of PANI-Gelidium, PANI-Codium, and PANI-Grateloupia.

\section{Conclusions}

We synthesised a series of PANI/sea algae as bio-composites. This technique is simple and conveniently to afford electrical conducting composites. Sea plants are flexible and have no stiff layer because they live beneath the water. The flexibility of the marine algae provides good affinity with PANI.

The marine algae have various natural materials on the surface, such as fucoidin [7]. Surface of the marine algae can play a role of reaction field. Combination of the materials from seaweeds and conducting polymers can be expected for new applications, such as flexible electrodes. Furthermore, marine algae have natural chemicals, which are valuable for medicine and chiral chemistry. Chemical reactions with the marine algae have possibilities of producing a new direction for development of organic materials with novel functions.

\section{Techniques}

IR spectra of the samples were obtained with a JASCO IR550. SEM observations performed with a JEOL JSM-7000F. ESR measurements were recorded on a Bruker EMX-T ESR spectrometer. Electrical conductivity was measured by four-probe method using Mitsubishi Chemical Analytech LORESTA-GP MCP-T610. 


\section{Acknowledgments}

We gratefully thank Prof. K. Inaba, Prof. S. Wada, Y. Tsuchiya, Y. Yamada, and Dr. D. Shibata (Shimoda Marine Centre, University of Tsukuba, Japan) for their kind instruction and warm support in the field-study and experiments at the Marine Centre. SEM observations were carried out at Microstructure Characterisation Platform Promotion Office, National Institute for Materials Science (NIMS). We thank Dr. Takeguchi (NIMS).

\section{References}

[1] G. G. Wallace, G. M. Spinks, L. A. P. Kane-Maguire, P. R. Teasdale, Conductive Electroactive polymers: Intelligent polymer systems, third edition. 2009, CRC Press, FL.

[2] I. Akatsuka, K. Iwamoto, Histochemical localization of agar and cellulose in the tissue of Gelidium Pacificum (Gelidiaceae, Rhodophyta), Botanica Marina 22 (1979) 367-370.

[3] A. Rahy, D. J. Yang, Synthesis of highly conductive polyaniline nanofibers. materials letters. 62 (2008) 4311-4314.

[4] D. Marsitzky, K. Mullen, 20 Years of "Synthetic Metals"- the role of synthesis, in: P. Bernier, S. Lefrant, G. Bidan (Eds), Advances in synthetic metals, twenty years of progress in science and technology, Elsevier Science SA, 1999, Switzerland, pp. 1-97.

[5] S. Stafström, J.L. Brédas, A.J. Epstein, H.S. Woo, D.B. Tanner, W.S. Huang, A.G. MacDiarmid, Polaron lattice in highly conducting polyaniline: Theoretical and optical studies, Phys. Rev. Lett. 59 (1987) 1464-1467.

[6] P. Petit, E. Jouguelet, J.E. Fischer, A.G. Rinzler, R.E. Smalley. Electron spin resonance and microwave resistivity of single-wall carbon nanotubes, Phys. Rev. B56 (1997) 9275-9278.

[7] Y. Goor, O. Goor, Y. Wollman, T. Chernichovski, D. Schwartz, S. Cabili, A. Iaina, Fucoidin, an inhibitor of leukocyte adhesion, exacerbates acute ischemic renal failure and stimulates nitric oxide synthesis. Scand. J. Urol. Nephrol.40 (2006) 57-62. 